\title{
Polymorph of 1,4-diketo-3,6-bis-(4'-dipyridyl)-pyrrolo-[3,4-c]pyrrole
}

\section{and their hydrogen bond network: A material for $\mathrm{H}_{2}$ gas sensor}

Jin Mizuguchi ${ }^{*}$, Tomohiko Imoda, Hiroo Takahashi ${ }^{* *}$ and H. Yamakami

Graduate School of Engineering, Yokohama National University

79-5 Tokiwadai, Hodogaya-ku, 240-8501 Yokohama, Japan

\begin{abstract}
Diketodiphenylpyrrolopyrroles (DPP) are industrially important red pigments. The title compound (DPPP) is a dipyridyl derivative that possesses a high proton affinity because of the $\mathrm{N}$ atoms of the pyridyl ring. A drastic change in shade as well as electrical conductivities by several orders of magnitude is observed due to protonation at the $\mathrm{N}$-atom. Therefore, DPPP has newly attracted attention as a material for $\mathrm{H}_{2}$ gas sensor. Then, we noticed that there are two crystal phases: one is quite sensitive to protons (phase I grown from the vapor phase) while the other is rather resistant to protonation (phase II recrystallized from solution). Therefore, structure analysis has been carried out in the present investigation in order to clarify the correlation between the structure and proton affinity. A striking difference is recognized in intermolecular hydrogen bonds between two phases: phase I is characterized only by $\mathrm{NH}^{\cdots} \mathrm{O}$ hydrogen bonds where two $\mathrm{N}$ atoms of the pyridyl rings remain free. On the other hand, phase II includes two types of the $\mathrm{H}$-bond based on $\mathrm{NH} \cdots \mathrm{O}$ and $\mathrm{NH} \cdots \mathrm{N}$ where the one $\mathrm{N}$ atom of the pyridyl ring is used by $\mathrm{NH} \cdots \mathrm{N}$ hydrogen bond and the other remains unused (i.e. free). Therefore, phase I is found to correspond to the phase which exhibits a high proton affinity while phase II is the one which is rather inactive for protons.
\end{abstract}

Keywords: diketopyrrolopyrrole, hydrogen gas sensor, crystal structure, electronic structure, polymorph

* Corresponding author. Tel. \& Fax: +81-45-339-3369

E-mail address: mizu-j@ynu.ac.jp

** Present address: Toyo Ink Engineering, Co. Ltd.

1-21-1Kaga, Itabashi, 173-8666 Tokyo, Japan 


\section{Introduction}

Diketodiphenylpyrrolopyrroles (for example DPP: Fig. 1(a)) are industrially important organic pigments used not only for paint industries as well as in the imaging areas [1]. DPPs have also attracted attention as a material useful for EL and color filters for LCD applications. The title compound (DPPP: Fig. 1(b)) is a dipyridyl derivative that shows a high proton affinity because of the $\mathrm{N}$ atom of the pyridyl ring [2]. Protonation at the $\mathrm{N}$ atom (shown in the inset of Fig.2) brings about a drastic color change from vivid red to violet, accompanied by changes in electrical conductivity and photoconduction [2]. For example, the absorption maximum is displaced from 560 to $580 \mathrm{~nm}$ due to protonation by vapor of nitric acid as shown in Fig.2 while the electrical resistivity reduces by several orders of magnitude. The proton affinity is obviously a negative factor for colorants because the color change easily occurs in pigment-dispersed layers due to gradual liberation of protons from the polymer matrix. The proton affinity is, however, a positive factor for applications for $\mathrm{H}_{2}$ gas sensors. For this reason, we have initiated the investigation for $\mathrm{H}_{2}$ gas sensors [3]. Then, we found that there exist two crystal phases in the solid state of DPPP: one is quite sensitive to protons (phase I: grown from the vapor phase) while the other exhibits a poor proton affinity (phase II: recrystallized from solution). We have successfully isolated these single crystals and analyzed the crystal structure of phases I and II in order to elucidate the correlation between the structure and proton affinity.

The present paper deals with the crystal and electronic structure of phases I and II of DPPP with special attention to their hydrogen bond network. 


\section{Experiment}

\subsection{Sample preparation and crystal growth}

DPPP was synthesized according to the method described in Ref.4 and purified six times by sublimation, using a two-zone furnace [5]. Single crystals of phase I were grown from the vapor phase in a closed system based on a two-zone furnace. On the other hand, single crystals of phase II were grown by recrystallization from solution in $N, N$-dimethylacetamide using an autoclave (Taiatsu Co. Ltd.; model TPR-2).

\subsection{Structure analysis}

Reflection data were collected by a R-AXIS RAPID-F diffractometer from Rigaku. Structure was solved by direct methods (SHELXS-86) and refinement was carried out by the least-squares method in teXsan program package.

\subsection{Polarized reflection spectra}

Polarized reflection spectra in the visible region were measured on single crystals by means of a microscope-spectrophotometer (UMSP 80 from Carl Zeiss) equipped with an R928 photomultiplier (HTV). Reflectivities were corrected relative to the reflection standard of silicon carbide.

\section{Results and discussion}

3.1 Crystallographic parameters and molecular conformation of DPPP 
Table 1 details the crystallographic parameters for phase I together with those of phase II [6]. In phase I, the crystal system is monoclinic and the space group is $P 2_{1} / n$. These are two molecules in the unit cell. On the other hand, the space group and molecular symmetry of phase II are $P 2_{1} / c$ and $C_{1}$, respectively. There are four molecules at the general positions in the unit cell.

Figs. 3(a) and 3(b) show the ORTEP plots of phases I and II, respectively. Because of the $C_{\mathrm{i}}$ symmetry in phase $\mathrm{I}$, the pyridyl rings are twisted in the same direction by about $6.5^{\circ}$ with respect to the heterocyclic ring. In phase II, the two pyridyl rings are asymmetrically twisted in opposite directions by $4.2^{\circ}$ and $15.3^{\circ}$.

\subsection{Molecular arrangement and intermolecular hydrogen bonds}

Figs. 4(a) and 4(b) shows the projection of the crystal structure of phase I onto the $(b, c)$ and $(a, c)$ planes, respectively. The dotted lines represent the intermolecular hydrogen bonds between the $\mathrm{NH}$ group of one molecule and the $\mathrm{O}$ atom of the neighboring one (N/O distance: $2.85 \AA$ ). There are chains of $\mathrm{NH}^{\cdots} \mathrm{O} \mathrm{H}$-bonds along the $b$-axis. This type of two-dimensional H-bond network is found in common in all DPP pigments. The molecules are arranged in a zigzag fashion along the $c$-axis and stacked along the $a$-axis. It is also to be noted that two $\mathrm{N}$ atoms in each pyridyl ring remain free and can accept protons. For this reason, phase $\mathrm{I}$ is ideal for applications of $\mathrm{H}_{2}$ gas sensors.

On the other hand, the H-bond fashion and H-bond network are quite different in phase II. The projection of the crystal structure onto the $(b, c)$ and $(a, c)$ planes is shown 
in Figs. 5(a) and 5(b), respectively. On the $(b, c)$ plane, there are two types of the $\mathrm{H}$-bond. One is the $\mathrm{NH} \cdots \mathrm{O}$ between the $\mathrm{NH}$ group of one molecule and the $\mathrm{O}$ atom of the neighboring one (N/O distance: $2.84 \AA$ ). The other is based on the $\mathrm{NH} \cdots \mathrm{N}$ between the $\mathrm{NH}$ group of one molecule and the $\mathrm{N}$ atom of the pyridyl ring of the neighboring molecule (N/N distance: $2.89 \AA$ ). In each molecule, there are two $\mathrm{H}$-bonds of the $\mathrm{NH} \cdots \mathrm{O}$ type as well as two bonds of the $\mathrm{NH} \cdots \mathrm{N}$ type. In addition, there remains one free $\mathrm{N}$ atom. The molecule-pairs which sit side by side through $\mathrm{NH} \cdots \mathrm{O}$ hydrogen bonds behave as the repeating unit in the crystal lattice and are arranged in a zigzag fashion along the $b$-axis as well as the $c$-axis on the molecular plane. There is also a local H-bond ring network composed of six molecules: four $\mathrm{NH}^{\cdots} \mathrm{N}$ and two bifurcated $\mathrm{NH} \cdots \mathrm{O}$ bonds. The present two-dimensional H-bond network is quite unique of phase II, not found in ordinary DPPs. The molecules are stacked along the $a$-axis. It should be also noted that in phase II, one $\mathrm{N}$ atom is used for the formation of $\mathrm{NH}^{\cdots \cdots} \mathrm{N}$ hydrogen bond while the other remains free. Therefore phase II is less sensitive to protons as compared with phase I. For this reason, phase II is appropriate for colorant applications because the color change caused by protonation is significantly suppressed.

\subsection{Polarized reflection spectra measured on single crystals}

Figs. 6(a) and 6(b) show the polarized reflection spectra of phase I measured on the $(b, c)$ plane of single crystals and the corresponding projection, respectively. The direction of the transition dipole as deduced from MO calculations is depicted in dotted lines in Fig.6(b). Polarized light was introduced in parallel or perpendicular to the $b$-axis. A prominent reflection band appears around $550 \mathrm{~nm}$ together with a reflection shoulder 
around $450 \mathrm{~nm}$ for polarization parallel to $b$-axis. On the other hand, these intense reflection bands completely disappear for polarization perpendicular to the $b$-axis. This indicates that the transition dipole points along the $b$-axis which is the direction of the $\mathrm{NH}^{\cdots} \mathrm{O}$ intermolecular H-bonds. The direction of the transition dipole as determined by experiment is in good agreement with that predicted by MO calculations.

Figs. 7(a) and 7(b) show the polarized reflection spectra of phase II measured on the (a,c) plane of single crystals and its corresponding projection, respectively. Measurements were made with polarized light along or perpendicular to the molecular plane. A strong reflection band appear around $450 \mathrm{~nm}$ together with small bands peaking around 480 and $540 \mathrm{~nm}$ for polarization along the molecular plane. These bands are, however, completely quenched for polarization perpendicular to the molecular plane.

As seen from the above results, it is remarkable to note that the reflection spectra for phases I and II are strikingly different, depending on the molecular arrangement governed by the fashion of the $\mathrm{NH}^{\cdots} \mathrm{O}$ and/or $\mathrm{NH}^{\cdots} \mathrm{N}$ hydrogen bond network. This is mainly due to excitonic interactions as discussed previously for a variety of DPP derivatives [7].

\section{Conclusion}

Structural characterization has been carried out on phases I and II in an attempt to clarify the correlation between the structure and proton affinity. The conclusions can be summarized as follows.

1. Phase I crystallizes in space group of $P 2_{1} / n$ with $Z=2$. There are four $N H \cdots O$ hydrogen bonds per molecule as found in ordinary DPPs. The $\mathrm{N}$ atom of the pyridyl 
ring remains free and thus can easily accept protons. Therefore, phase I is ideal for applications of $\mathrm{H}_{2}$ gas sensors. The transition dipole of phase I points along the $\mathrm{NH} \cdots \mathrm{O}$ intermolecular hydrogen bond.

2. In phase II, the space group is $P 2_{1} / c$ with $Z=4$ and there are two types of hydrogen bonds: $\mathrm{NH} \cdots \mathrm{O}$ and $\mathrm{NH}^{\cdots} \cdots \mathrm{N}$. The $\mathrm{NH}^{\cdots} \cdots \mathrm{N}$ hydrogen bond is formed between the $\mathrm{NH}$ group of one molecule and the $\mathrm{N}$ atom of the pyridyl ring of the neighboring molecule. Then, one of the two $\mathrm{N}$ atoms of the pyridyl rings is protected by the $\mathrm{NH} \cdots \mathrm{N}$ hydrogen bond while the other is free. Therefore, phase II is rather resistant to color change due to protonation. 


\section{References}

[1] Herbst M, Hunger, K. Industrial Organic Pigments, Weinheim: VCH; 1993.

[2] Mizuguchi J. Ber Bunsenges Phys Chem 1993; 97: 684.

[3] Takahashi H, Mizuguchi J. submitted to Sensors and Actuators B.

[4] Rochat A. C, Cassar L, Iqbal, A. U S Pat 4, 579, 949; 1986.

[5] Mizuguchi J. Krit Tech 1981; 16: 695.

[6] Mizuguchi J, Takahashi H, Yamakami, H. Z Kristallogr NCS 2002; 217: 519 .

[7] Mizuguchi J. J Phys Chem A 2000; 104: 1817. 
Figure captions:

Fig.1 Molecular conformation: (a) DPP and (b) DPPP.

Fig.2 Solid-state spectra of evaporated DPPP before and after protonation with the vapor of nitric acid.

Fig.3 ORTEP plots: (a) phase I and (b) phase II.

Fig.4 Projection of phase I onto: (a) $(b, c)$ plane and (b) $(a, c)$ plane.

Fig.5 Projection of phase II onto: (a) $(b, c)$ plane and (b) $(a, c)$ plane.

Fig.6 (a) Polarized reflection spectra of phase I measured on the (100) plane of single crystals and (b) projection of phase I onto the $(b, c)$ plane.

Fig.7 (a) Polarized reflection spectra of phase II measured on the (010) plane of single crystals and (b) projection of phase II onto the $(a, c)$ plane. 
Table 1

Crystallographic parameters for phases I and II of DPPP

\begin{tabular}{ccc}
\hline & Phase I & Phase II \\
\hline Crystal system & monoclinic & monoclinic \\
Space group & $P 21 / n$ & $P 21 / c$ \\
Molecular symmetry & $C_{\mathrm{i}}$ & $C_{1}$ \\
$Z$ & 2 & 4 \\
$a(\AA)$ & $3.722(1)$ & $3.695(1)$ \\
$b(\AA)$ & $6.263(3)$ & $18.201(2)$ \\
$c(\AA)$ & $26.506(9)$ & $18.456(2)$ \\
$\beta\left({ }^{\circ}\right)$ & $94.41(2)$ & $94.68(1)$ \\
$\mathrm{V}\left(\AA^{3}\right)$ & $616.0(4)$ & $1237.1(3)$ \\
\hline
\end{tabular}

(a)<smiles>O=C1NC(c2ccccc2)=C2C(=O)NC(c3ccccc3)=C12</smiles>

(b)<smiles>O=C1NC(c2ccncc2)=C2C(=O)NC(c3ccncc3)=C12</smiles>

Fig. 1

(a)

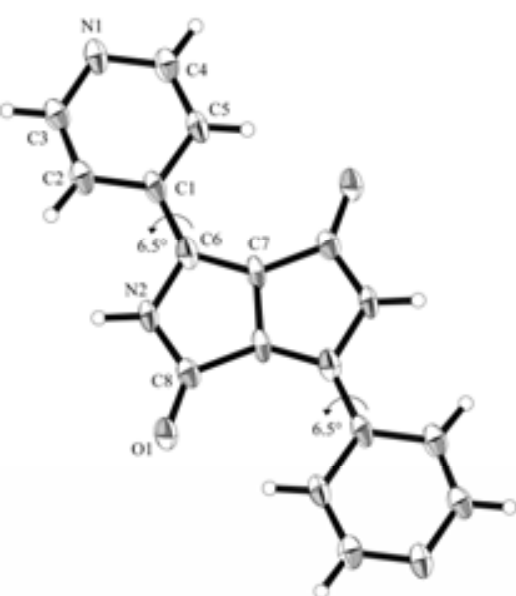

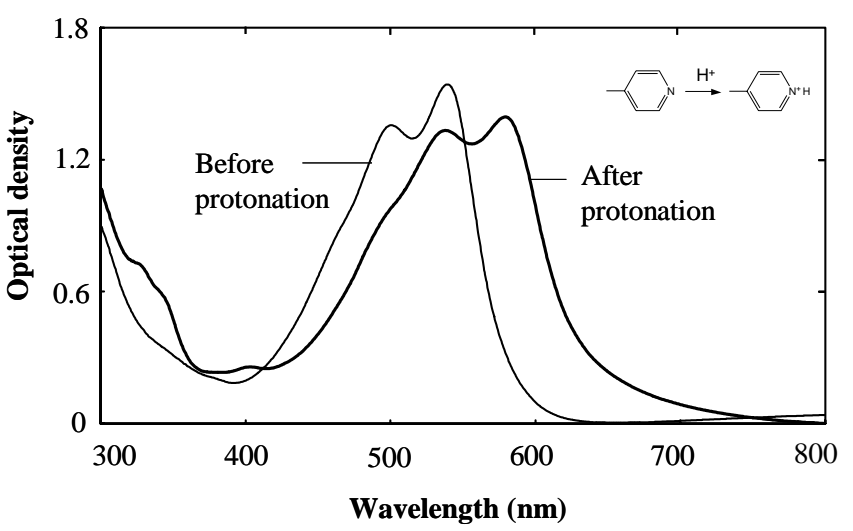

Fig. 2

(b)

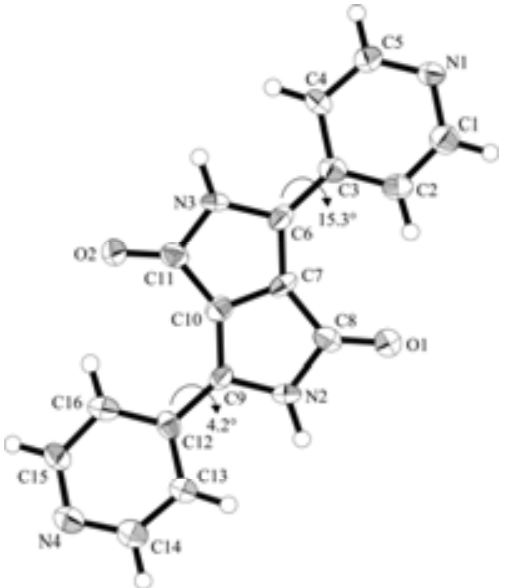

Fig. 3 
(a)

(b)

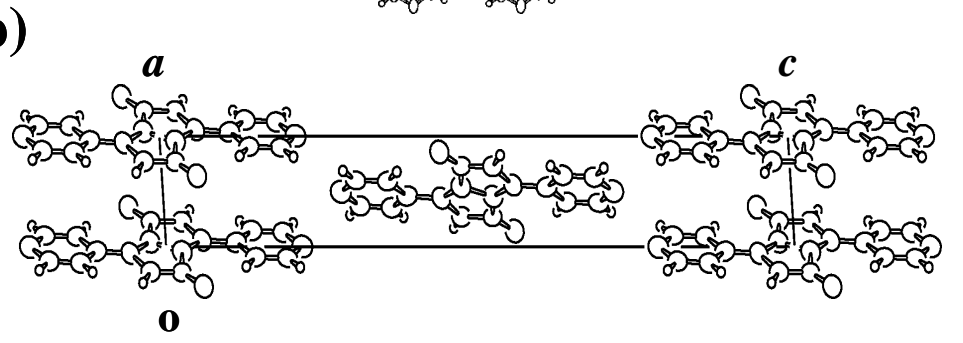

Fig. 4

(a)

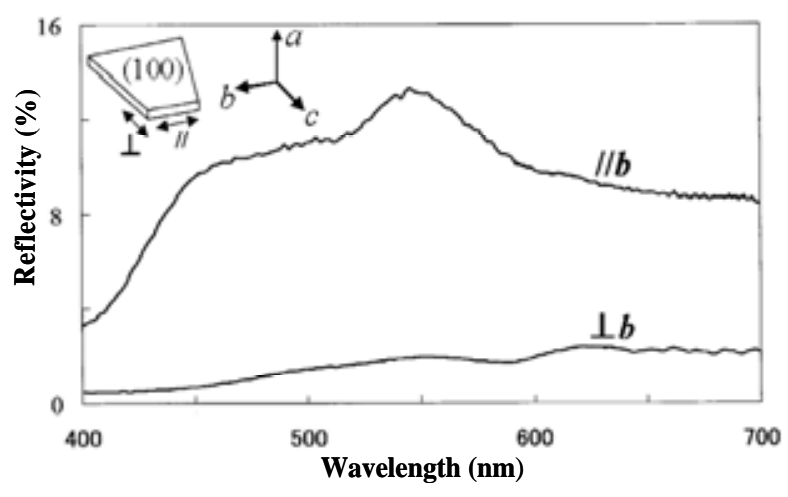

(b)

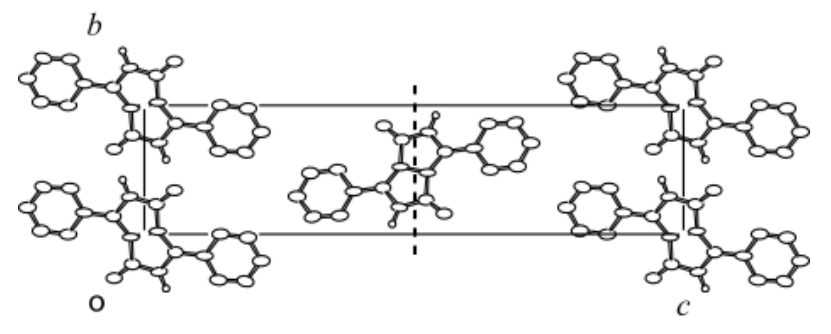

-....-. Transition dipole

Fig. 6 (a)

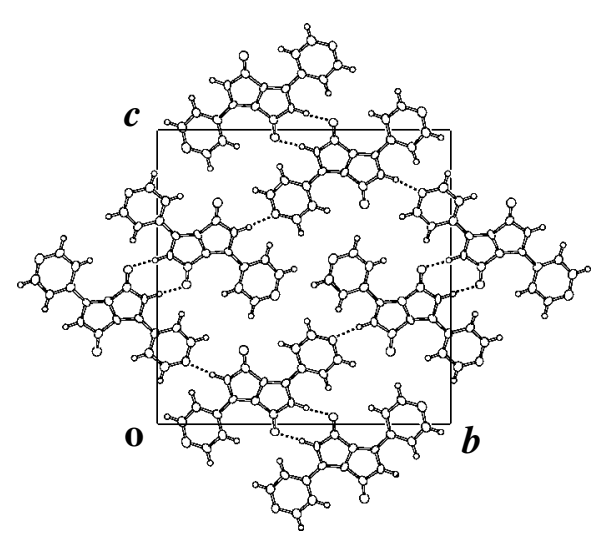

(b)

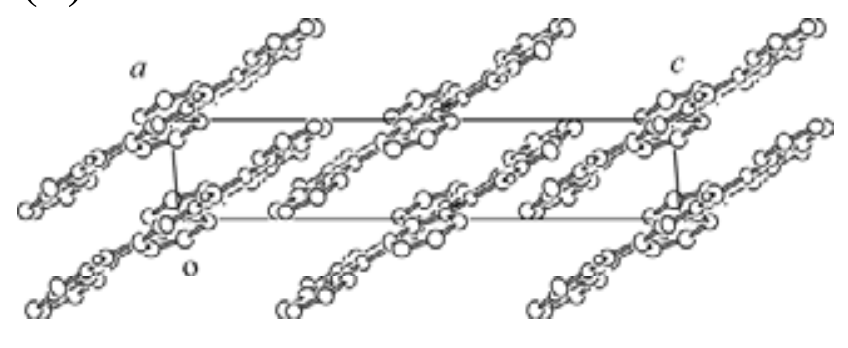

Fig. 5

(a)

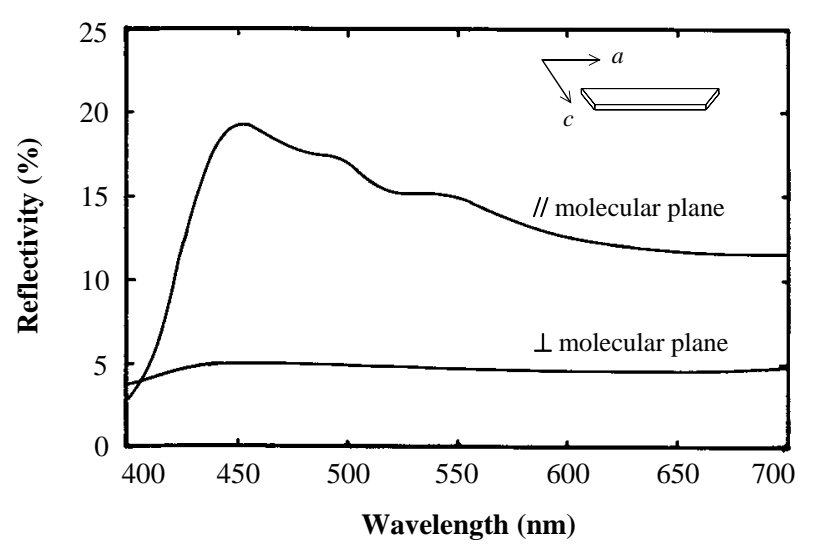

(b)

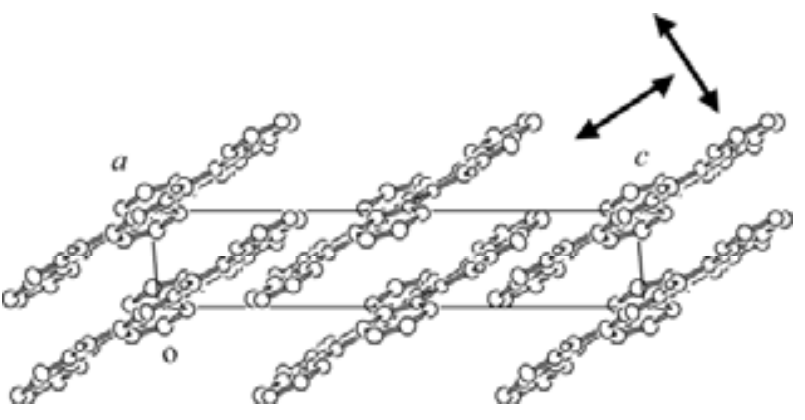

Fig. 7 